\title{
Dealing with the Unknown: Metabolomics and Metabolite Atlases
}

\author{
Benjamin P. Bowen and Trent R. Northen \\ Department of GTL Bioenergy and Structural Biology, Life Sciences Division, Lawrence Berkeley National \\ Laboratory, Berkeley, California, USA \\ Metabolomics is the comprehensive profiling of the small molecule composition of a biological \\ sample. Since metabolites are often the indirect products of gene expression, this approach is being \\ used to provide new insights into a variety of biological systems (clinical, bioenergy, etc.). A grand \\ challenge for metabolomics is the complexity of the data, which often include many experimental \\ artifacts. This is compounded by the tremendous chemical diversity of metabolites. Identification \\ of each uncharacterized metabolite is in many ways its own puzzle (compared with proteomics, \\ which is based on predictable fragmentation patterns of polypeptides). Therefore, effective data \\ reduction/prioritization strategies are critical for this rapidly developing field. Here we review \\ liquid chromatography electrospray ionization mass spectrometry (LC/MS)-based metabolomics, \\ methods for feature finding/prioritization, approaches for identifying unknown metabolites, and \\ construction of method specific 'Metabolite Atlases'. (J Am Soc Mass Spectrom 2010, 21, \\ 1471-1476) () 2010 Published by Elsevier Inc. on behalf of American Society for Mass Spectrometry
}

$\mathrm{N}$ ature utilizes a tremendous diversity of metabolites, and it is estimated that there are $>200,000$ plant metabolites alone [1]. Metabolomics is a rapidly growing field for studying the small molecule composition of a biological system. Liquid chromatography coupled to electrospray ionization mass spectrometry (LC/MS) is becoming a method of choice for profiling metabolites in complex biological samples. This is due to its ability to effectively ionize a breath of metabolites with minimal fragmentation (versus gas chromatography-mass spectroscopy (GC/MS), robustness, and ability to scale-up to support tandem mass spectrometry based structural studies (versus capillary electrophoresis-mass spectrometry (CE-MS)) [2]. However, known metabolites are typically a small portion of the data obtained in a LC/MS metabolomics experiment $(\sim 10 \%)$ [3] where the bulk are MS-artifacts and uncharacterized metabolites.

Identification of unknown metabolites is cost and effort intensive, often requiring preparative scale isolation for nuclear magnetic resonance (NMR) studies or extensive chemical synthesis to enable structural comparisons using tandem mass spectrometry (MS/MS) [4]. Therefore, it is not surprising that the majority of reports focus on changes in metabolites that have authentic standards or at a minimum are found in metabolite databases. Yet, there are only a few thousand commercially available analytical standards due to lack of demand and the inherent instability of many metabolites. These standards and the endogenous me-

Address reprint requests to Dr. T. R. Northen, Department of GTL Bioenergy and Structural Biology, Life Sciences Division, Lawrence Berkeley National Laboratory, 1 Cyclotron Road, Berkeley, CA 94720, USA. E-mail: TRNorthen@lbl.gov tabolites contained in databases represent only a portion of endogenous metabolites. Therefore, effective methods for prioritizing, studying, and ultimately identifying uncharacterized metabolites is a critical development for LC/MS based metabolomics [5-10]. Here we discuss current LC/MS metabolomic approaches (detailed elsewhere $[2,4,11,12])$ and their integration into 'Metabolite Atlases' to facilitate annotation and prioritization of uncharacterized metabolites.

\section{LC/MS Based Metabolomics}

The conventional metabolomics workflow is summarized in Figure 1. At the heart of any mass spectrometry experiment is the generation of gas-phase ions. Metabolomics experiments primarily utilize electrospray ionization (ESI) or electron ionization (EI). Each has its advantage, EI effectively ionizes and fragments most volatile molecules, and these fragments are easily compared with large spectral databases to aid identification. However, ESI is often preferred for profiling 'unknown' metabolites since this 'soft' desorption/ionization approach efficiently forms 'intact' molecular ions that aids initial identification of unknowns. This coupled with the high mass accuracy of many instruments $(<5 \mathrm{ppm})$ facilitates the initial ('putative') identification of metabolites by searching metabolite databases [13]. This approach has been implemented in a wide range of applications primarily related to functional genomics and clinical studies $[4,14]$.

The number of metabolites detected can be increased by chromatographic methods (i.e., reverse and normal phase chromatography). EI is typically coupled to gas chromatography (GC/MS), and ESI is often coupled to 

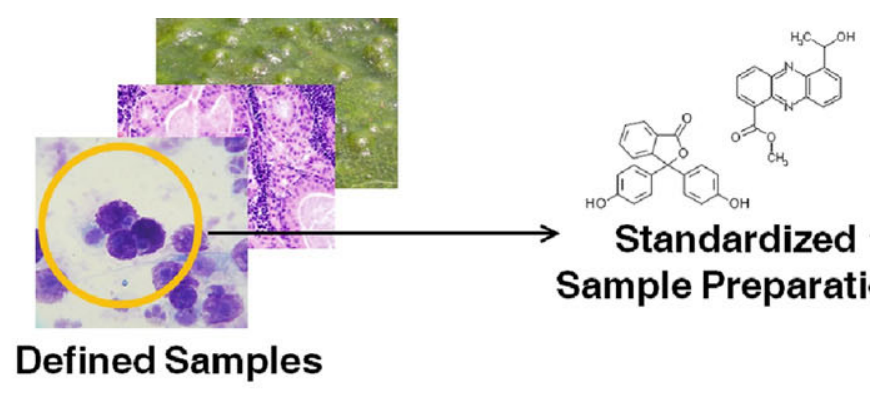
Sample Preparation

Defined Samples

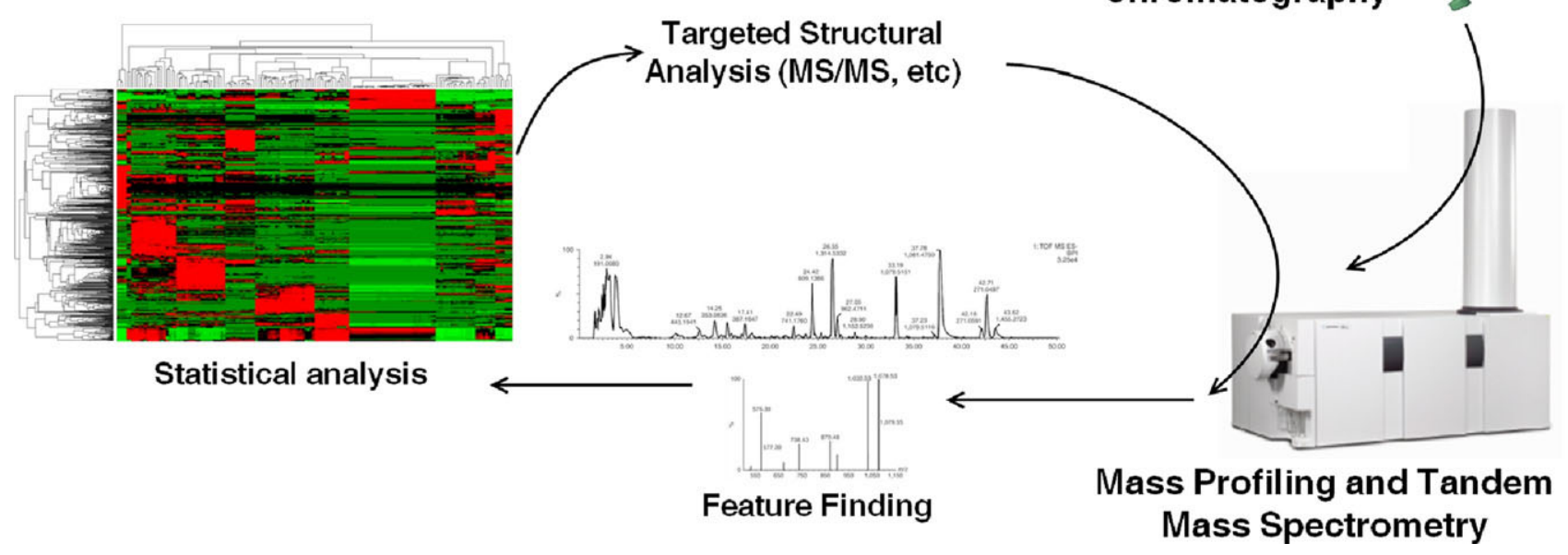

Figure 1. Overview a typical LC/MS metabolomic workflow. This includes sample preparation steps (quenching, cell lysis, extraction, etc.), LC/MS profiling, feature finding, statistical analysis, and concludes with structural confirmation of unknown metabolites.

liquid chromatography (LC/MS) or capillary electrophoresis (CE-MS) to resolve complex mixtures. Though it should be noted that Fourier transform ion cyclotron resonance mass spectrometry (FTICR/MS) can often resolve complex mixtures of metabolites without chromatography [15]. The wide range of chromatography (LC) options allow physical separation of most molecules to reduce signal suppression [16]. While reversephase methods tend to utilize C18 columns, a wide range of normal phase materials are utilized $[16,17]$ e.g., popular new materials, including hydrophilic interaction chromatography (HILIC) and aqueous normal phase (ANP) have recently been developed for improved separation of polar metabolites [18].

The combination of exact mass measurements and LC retention time are used to define chromatographic/ ion features $(m / z, R T)$ that are taken as a first approximation of a molecular identifier. Higher specificity features are obtained through the addition of fragmentation data $(\mathrm{m} / \mathrm{z}$ parent, RT, $\mathrm{m} / \mathrm{z}$ daughters). However, in practice, it is difficult to obtain useful fragmentation data for all features in an untargeted profiling experiment and it is therefore useful to utilize preferred or target lists for features of interest.

There are a variety of algorithms for defining these 'features' and comparing them with metabolite databases as discussed in more detail below. A limitation of defining unidentified ions as features is that both dimensions $(m / z, R T)$ are method-dependent. Changes in the LC method, mobile phase, biological matrix, etc. can result in a new RT for the same ion or new ions (adducts). Changes to the ionization source (i.e., fragmentor and capillary voltages) will change the types/ abundances of ions (adducts and neutral losses). Further, experimental variables such as the extraction method can have dramatic effects, requiring careful experimentation to obtain comprehensive and reproducible results $[19,20]$.

Since features depend on the experimental details, it is valuable to standardize metabolomics methods and metabolomics data reporting. This can be accomplished by following the reporting standards defined by the metabolomics community [21-23]. For example, experimental details should be linked to the metabolite features, which can also be linked to metabolite databases, i.e., METLIN [5], KEGG [6], MetaCyc [7], the Golm Metabolome Database [8], HMDB [9], LIPIDMAPS [10], and MassBank (URL: http:/ / www.massbank. $\mathrm{jp} /$ ) to facilitate identification. In many ways, these efforts follow in the footsteps of other systems approaches (genomics, transcriptomics, and proteomics) that have established mechanisms for data-sharing and analysis [24]. 


\section{Feature Finding and Prioritization}

Due to the cost and effort required to identify unknown metabolites, only the 'most important' features are selected for identification. Generally, efforts entail four steps: first, the unique features for each sample are computationally defined to allow comparison of abundance across samples in an experiment. Second, statistical methods are used to identify the largest and most significantly changing features. Third, identified features are annotated to assign isotopes, adducts, degradation products, etc. Finally, the resulting neutral masses are searched against metabolite databases to identify candidates for further analysis.

In the first step, data files must be processed by algorithms capable of identifying features that result from the various signatures of an ionized metabolite. Again, a feature is simply a collection of data points or a region in $m / z$ and time. In principle, the feature will have a chromatogram that is Gaussian in shape, a well defined peak, and the distribution of data points in the feature will be narrowly distributed at a given $\mathrm{m} / \mathrm{z}$. In practice, the characteristic shape of a metabolite feature can be highly varied. Features are defined using a wide array of existing algorithms, such as XCMS [25], msInspect [26], mzMine [27], commercial options, and many more [28, 29]. As an open-source application, XCMS is the most widely used of these algorithms, and the recently described CentWave peak finding strategy is an important development [30].

To prioritize features for subsequent identification, statistical methods based on data clustering, dimensional reduction, and multiple hypothesis testing are used. In addition to parametric and nonparametric hypothesis tests, these include principal component analysis (PCA) [31], partial-least-squares discriminant analysis (PLS-DA) [3], and self organizing networks (GEDI) [32]. These tools can reduce the complexity of data by allowing researchers to focus efforts on groups of features that exhibit similar abundance patterns. One commonly used, freely available statistical package that enables a wide variety of data analyses is MultiExperiment Viewer $(\mathrm{MeV})$ [33]. This application is widely used for the analysis of microarray data and has several features making it an excellent choice for metabolomics data analysis.

Once compound specific features are defined and annotated, the accurate neutral masses of these compounds can be searched in a variety of metabolite databases to create a list of putative identifications. The reader is referred to a recent review for additional discussion on metabolite databases [34]. However, there are limitations to this approach. The databases are not comprehensive and many contain a wide variety of unnatural metabolites [35].

Recent works illustrate the predominant workflows for untargeted metabolomic profiling experiments: Wikoff et al. used LC/MS and XCMS to analyze the cerebrospinal fluid of SIV-infected macaques [36]. Unequal-variance $t$-tests and fold-change between the infected and uninfected samples was used to reduce $>3000$ features to the 12 most important. These were then identified using authentic standards and tandem mass spectrometry. In another study, Tyburski et al. used LC/MS metabolomics to study the response of mice to $\gamma$-radiation exposure [37]. MarkerLynx was used for feature identification. Self-Organizing Maps, PCA, and PLS techniques were used to prioritize the features affected which were subsequently identified by comparing their fragmentation patterns with authentic standards. These examples and others have been utilized within the framework of untargeted metabolomics [38]. In each case, definitive identification of a few uncharacterized metabolites represents a major research effort.

\section{Current Approaches for Identifying Unknown Metabolites}

The development of MS/MS spectral databases such as METLIN and MassBank are of great assistance in the identification of unknown metabolites. One concern of this approach is the consistency of fragmentation patterns between instruments. Hence, it is a good practice to obtain the authentic standard for final identification. Unfortunately, many metabolites are neither in spectral databases nor commercially available, making identification of these an arduous process. The higher sensitivity of LC/MS versus NMR typically requires either isolation/enrichment for direct structural analysis using NMR, or further mass spectrometry based structural analysis [39]. If sufficient sample is available, enrichment may be the most direct route. However, in many cases it is not possible to separate sufficient quantities due to sample availability and/or complexity. In these cases, it is necessary to synthesize libraries of related hypothetical molecules and compare features (e.g., $m / z$, fragmentation masses, and method specific retention time) with the unknown metabolite [37, 40].

There are a number of approaches to obtain less definitive structural identification. Detection of diagnostic fragmentation patterns is often a preferred method [41]. Hence, the further development of LC/MS and MS/MS databases and search algorithms are an important goal for simplifying this process as are computational tools for predicting structure based on MS/MS data [42]. Stable isotopic label incorporation or chemical derivatization can also aid in structural confirmation based on mass shifts in specific fragments, though this approach is not viable for many experimental situations (i.e., clinical samples) [2, 43]. For example, if it is postulated that a metabolite contains a nitrogen, comparison of the metabolite from ${ }^{14} \mathrm{~N}$ versus ${ }^{15} \mathrm{~N}$ cultures can confirm the number of nitrogen atoms per molecule and link them to specific MS/MS fragments. It can also be useful to globally compare isotopically labeled and unlabeled metabolite pools [44-46]. Mass shifts between labeled and unlabeled metabolites 
Single Experiments

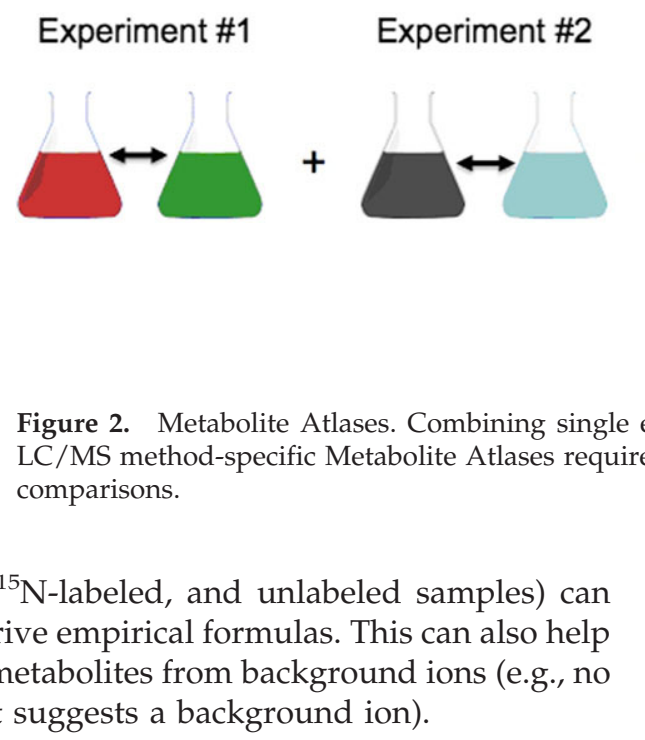

$\left({ }^{13} \mathrm{C}\right.$-labeled, ${ }^{15} \mathrm{~N}$-labeled, and unlabeled samples) can be used to derive empirical formulas. This can also help differentiate metabolites from background ions (e.g., no chemical shift suggests a background ion).

\section{Construction of Method-Specific Metabolite Atlases}

Construction of Metabolite Atlases is simply a matter of accumulating data from multiple experiments performed on similar biological matrices (samples) using identical LC/MS methods (Figure 2). This allows researchers to leverage the same annotations across multiple experiments. For example 'artifact' features (i.e., adducts and degradation products) can be identified once, and in the long-term, this avoids wasted effort re-identifying nonbiological features. This approach has the risk that some features are in fact different compounds in the various experiments. Therefore, it is a good practice to confirm instrument calibration, retention time stability, and perform tandem mass spectrometry to confirm that compounds are identical before drawing any conclusions.

Adducts can be annotated by linking features with common mass transformations (Figure 3) [47]. Specifically, retention time, MS/MS, and mass-difference in- formation can be utilized to provide a signature for a particular metabolite that is detected as multiple features due to isotopes and experimental artifacts (adducts, neutral loss, etc.). There are computational tools that can assist in this process to help determine the neutral mass of the compound [48-50]. This information is then used to annotate possible chemical formulas (including those identified using stable isotope labels) and information from metabolite databases (i.e., KEGG identifiers and names). Annotations should also include experimental details to allow subsequent comparisons across experiments (Figure 2).

The Atlas also associates features that exhibit similar patterns of abundance as shown in Figure 3. Correlationbased relationships retrieve metabolite features that are significantly different between specified samples. These can also be correlated with protein-abundance, sample types, or some other meta-data in the specified samples. While a feature may not be important in the context of a single experiment, it may be found to be important across multiple experiments. In some cases it may be observed that unknown features are correlated with known metabolites, which can suggest biological associations. This point is illustrated in a recent study by Wren [51], where the Gene Expression Omnibus (GEO)

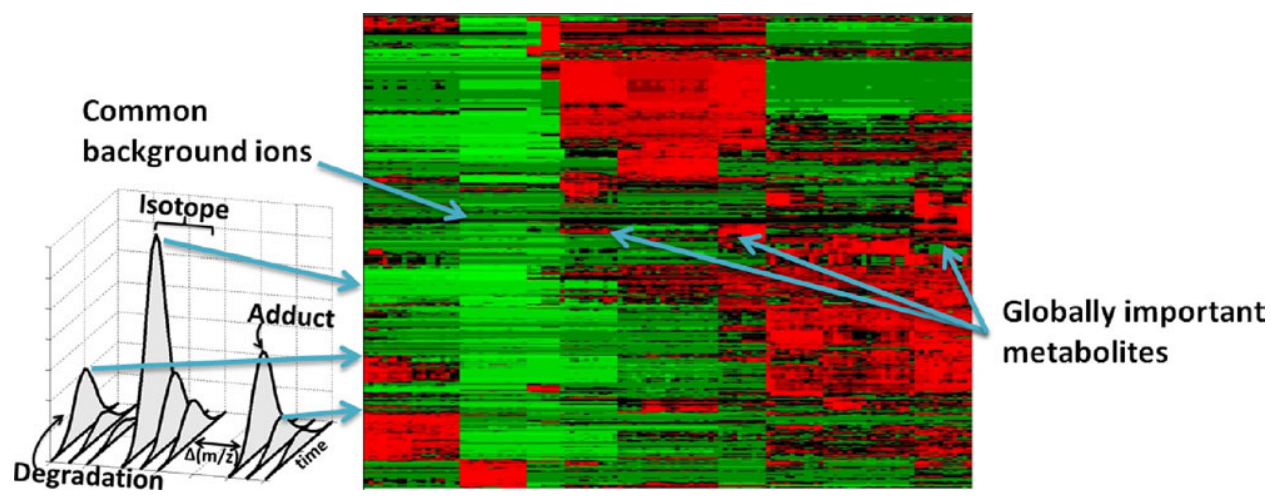

Figure 3. Experimental and higher order analysis of Metabolite Atlases. This format allows feature annotations (identified compounds, adducts, isotopes, neutral losses, etc.) to be leveraged across multiple experiments and can reveal common background ions and prioritize new features for further characterization. 
was used to correlate expression patterns to create new functional associations for uncharacterized transcripts. These types of complex queries illustrate the power and utility of data integration across experiments.

\section{Summary}

The extent to which researchers can select the most critical features governs the risk in making the significant effort required to identify them. It is therefore not surprising that the metabolomics community has invested major efforts in developing approaches for the identification of uncharacterized metabolites from single experiments. Moving beyond these to more global analysis requires implementation of data standards, reporting standards, new computational tools, and the further development of metabolite databases. Research groups carrying out large-scale metabolomics experiments can place their findings into a broader context by constructing method-specific Metabolite Atlases. These can then be used for annotation purposes to simplify feature annotation and enable multi-experiment analysis.

\section{Acknowledgments}

The authors gratefully acknowledge support from the Department of Energy [DE-AC02-05CH11231] and from the California Breast Cancer Research Program [15IB-0063]. They also thank Richard Baran, Chris Petzold, and Paul Wilmes for useful comments.

\section{References}

1. Fiehn, O. Metabolomics-The Link Between Genotypes and Phenotypes. Plant Mol. Biol. 2002, 48, 155-171.

2. Dettmer, K.; Aronov, P. A.; Hammock, B. D. Mass Spectrometry-Based Metabolomics. Mass Spectrom. Rev. 2007, 26, 51-78.

3. Jansson, J.; Willing, B.; Lucio, M.; Fekete, A.; Dicksved, J.; Halfvarson, J.; Tysk, C.; Schmitt-Kopplin, P. Metabolomics Reveals Metabolic Biomarkers of Crohn's Disease. PLoS ONE. 2009, 4, e6386.

4. Ceglarek, U.; Leichtle, A.; Brügel, M.; Kortz, L.; Brauer, R.; Bresler, K.; Thiery, J.; Fiedler, G. Challenges and Developments in Tandem Mass Spectrometry-Based Clinical Metabolomics. Mol. Cell. Endocrinol. 2009, $301,266-271$.

5. Smith, C. A.; O'Maille, G.; Want, E. J.; Qin, C.; Trauger, S. A.; Brandon, T. R.; Custodio, D. E.; Abagyan, R.; Siuzdak, G. METLIN-A Metabolite Mass Spectral Database. Therapeutic Drug Monitoring 2005, 27, 747-751.

6. Kanehisa, M.; Goto, S. KEGG: Kyoto Encyclopedia of Genes and Genomes. Nucleic Acids Res. 2000, 28, 27-30.

7. Caspi, R.; Foerster, H.; Fulcher, C.; Kaipa, P.; Krummenacker, M.; Latendresse, M.; Paley, S.; Rhee, S.; Shearer, A.; Tissier, C. The MetaCyc Database of Metabolic Pathways and Enzymes and the BioCyc Collection of Pathway/Genome Databases. Nucleic Acids Res. 2008, 36, D623.

8. Kopka, J.; Schauer, N.; Krueger, S.; Birkemeyer, C.; Usadel, B.; Bergmuller, E.; Dormann, P.; Weckwerth, W.; Gibon, Y.; Stitt, M.; Willmitzer, L.; Fernie, A. R.; Steinhauser, D. Gmd@Csb.Db: The Golm Metabolome Database. Bioinformatics 2005, 21, 1635-1638.

9. Wishart, D.; Tzur, D.; Knox, C.; Eisner, R.; Guo, A.; Young, N.; Cheng, D.; Jewell, K.; Arndt, D.; Sawhney, S. HMDB: The Human Metabolome Database. Nucleic Acids Res. 2007, 35 (Database Issue), D521-D526.

10. Fahy, E.; Subramaniam, S.; Brown, H. A.; Glass, C. K.; Merrill, A. H.; Murphy, R. C.; Raetz, C. R. H.; Russell, D. W.; Seyama, Y.; Shaw, W.; Shimizu, T.; Spener, F.; van Meer, G.; VanNieuwenhze, M. S.; White, S. H.; Witztum, J. L.; Dennis, E. A. A Comprehensive Classification System for Lipids. J. Lipid Res. 2005, 46, 839-861.

11. Goodacre, R.; Vaidyanathan, S.; Dunn, W.; Harrigan, G.; Kell, D. Metabolomics by Numbers: Acquiring and Understanding Global Metabolite Data. Trends Biotechnol. 2004, 22, 245-252.

12. Chen, C.; Gonzalez, F.; Idle, J. LC-MS-Based Metabolomics in Drug Metabolism. Drug Metab. Rev. 2007, 39, 581-597.

13. Prakash, C.; Shaffer, C.; Nedderman, A. Analytical Strategies for Identifying Drug Metabolites. Mass Spectrom. Rev. 2007, 26, 340-369.

14. Baran, R.; Reindl, W.; Northen, T. Mass Spectrometry Based Metabolomics and Enzymatic Assays for Functional Genomics. Curr. Opin. Microbiol. 2009, 12, 547-552.
15. Jain, M.; Petzold, C. J.; Schelle, M. W.; Leavell, M .D.; Mougous, J. D.; Bertozzi, C. R.; Leary, J. A.; Cox, J. S. Lipidomics Reveals Control of Mycobacterium tuberculosis Virulence Lipids Via Metabolic Coupling. Proc. Natl. Acad. Sci U.S.A. 2007, 104, 5133-5138.

16. Garcia, D. E.; Baidoo, E. E.; Benke, P. I.; Pingitore, F.; Tang, Y. J.; Villa, S.; Keasling, J. D. Separation and Mass Spectrometry in Microbial Metabolomics. Curr. Opin. Microbiol. 2008, 11, 233-239.

17. Bajad, S. U.; Lu, W.Y.; Kimball, E. H.; Yuan, J.; Peterson, C.; Rabinowitz, J. D. Separation and Quantitation of Water Soluble Cellular Metabolites by Hydrophilic Interaction Chromatography-Tandem Mass Spectrometry. J. Chromatogr. A 2006, 1125, 76-88.

18. Pesek, J. J.; Matyska, M. T.; Hearn, M. T. W.; Boysen, R. I. Aqueous Normal-Phase Retention of Nucleotides on Silica Hydride Columns. J. Chromatogr. A 2009, 1216, 1140-1146.

19. Want, E.; O'Maille, G.; Smith, C.; Brandon, T.; Uritboonthai, W.; Qin, C.; Trauger, S.; Siuzdak, G. Solvent-Dependent Metabolite Distribution, Clustering, and Protein Extraction for Serum Profiling with Mass Spectrometry. Anal. Chem. 2006, 78, 743-752.

20. Crews, B.; Wikoff, W. R.; Patti, G. J.; Woo, H. K.; Kalisiak, E.; Heideker, J.; Siuzdak, G. Variability Analysis of Human Plasma and Cerebral Spinal Fluid Reveals Statistical Significance of Changes in Mass SpectrometryBased Metabolomics Data. Anal. Chem. 2009, 81, 8538-8544.

21. Jenkins, H.; Hardy, N.; Beckmann, M.; Draper, J.; Smith, A.; Taylor, J.; Fiehn, O.; Goodacre, R.; Bino, R.; Hall, R. A Proposed Framework for the Description of Plant Metabolomics Experiments and Their Results. Nat. Biotechnol. 2004, 22, 1601-1606.

22. Fiehn, O.; Wohlgemuth, G.; Scholz, M.; Kind, T.; Lee, D.; Lu, Y.; Moon, S.; Nikolau, B. Quality Control for Plant Metabolomics: Reporting MSI-Compliant Studies. Plant J. 2008, 53, 691-704.

23. Sumner, L. W.; Amberg, A.; Barrett, D.; Beale, M. H.; Beger, R.; Daykin, C. A.; Fan, T. W. M.; Fiehn, O.; Goodacre, R.; Griffin, J. L.; Hankemeier, T.; Hardy, N.; Harnly, J.; Higashi, R.; Kopka, J.; Lane, A .N.; Lindon, J. C.; Marriott, P.; Nicholls, A. W.; Reily, M. D.; Thaden, J. J.; Viant, M. R. Proposed Minimum Reporting Standards for Chemical Analysis. Metabolomics 2007, 3, 211-221.

24. Brazma, A.; Hingamp, P.; Quackenbush, J.; Sherlock, G.; Spellman, P. Stoeckert, C.; Aach, J.; Ansorge, W.; Ball, C. A.; Causton, H. C.; Gaasterland, T.; Glenisson, P.; Holstege, F. C. P.; Kim, I. F.; Markowitz, V.; Matese, J. C.; Parkinson, H.; Robinson, A.; Sarkans, U.; SchulzeKremer, S.; Stewart, J.; aylor, R.; Vilo, J.; Vingron, M. Minimum Information About a Microarray Experiment (MIAME)-Toward Standards for Microarray Data. Nat. Genet. 2001, 29, 365-371.

25. Smith, C. A.; Want, E. J.; O'Maille, G.; Abagyan, R.; Siuzdak, G. XCMS: Processing Mass Spectrometry Data for Metabolite Profiling Using Nonlinear Peak Alignment, Matching, and Identification. Anal. Chem. 2006, 78, 779-787.

26. Bellew, M.; Coram, M.; Fitzgibbon, M.; Igra, M.; Randolph, T.; Wang, P.; May, D.; Eng, J.; Fang, R. H.; Lin, C. W.; Chen, J. Z.; Goodlett, D.; Whiteaker, J.; Paulovich, A.; McIntosh, M. A Suite of Algorithms for the Comprehensive Analysis of Complex Protein Mixtures Using HighResolution LC-MS. Bioinformatics 2006, 22, 1902-1909.

27. Katajamaa, M.; Oresic, M. Processing Methods for Differential Analysis of LC/MS Profile Data. BMC Bioinformatics 2005, 6, 179.

28. Katajamaa, M.; Orešič, M. Data Processing for Mass SpectrometryBased Metabolomics. J. Chromatogr. A 2007, 1158, 318-328.

29. Werner, E.; Croixmarie, V.; Umbdenstock, T.; Ezan, E.; Chaminade, P. Tabet, J.; Junot, C. Mass Spectrometry-Based Metabolomics: Accelerating the Characterization of Discriminating Signals by Combining Statistical Correlations and Ultrahigh Resolution. Anal. Chem. 2008, 80, $4918-4932$.

30. Tautenhahn, R.; Bottcher, C.; Neumann, S. Highly Sensitive Feature Detection for High Resolution LC/MS. BMC Bioinformatics 2008, 9, 504

31. Taylor, J.; King, R. D.; Altmann, T.; Fiehn, O. Application of Metabolomics to Plant Genotype Discrimination Using Statistics and Machine Learning. Bioinformatics 2002, 18, S241-S248.

32. Patterson, A. D.; Li, H.; Eichler, G. S.; Krausz, K. W.; Weinstein, J. N. Formace, A. J.; Gonzalez, F. J.; Idle, J. R. UPLC-ESI-TOFMS-Based Metabolomics and Gene Expression Dynamics Inspector Self-Organizing Metabolomic Maps as Tools for Understanding the Cellular Response to Ionizing Radiation. Anal. Chem. 2008, 80, 665-674.

33. Hirayama, A.; Kami, K.; Sugimoto, M.; Sugawara, M.; Toki, N.; Onozuka, H.; Kinoshita, T.; Saito, N.; Ochiai, A.; Tomita, M.; Esumi, H.; Soga, T. Quantitative Metabolome Profiling of Colon and Stomach Cancer Microenvironment by Capillary Electrophoresis Time-of-Flight Mass Spectrometry. Cancer Res. 2009, 69, 4918-4925.

34. Tohge, T.; Fernie, A. R. Web-Based Resources for Mass-SpectrometryBased Metabolomics: A User's Guide. Phytochemistry 2009, 70, 450-456.

35. Kind, T.; Scholz, M.; Fiehn, O. How Large is the Metabolome? A Critical Analysis of Data Exchange Practices in Chemistry. PLoS ONE. 2009, 4, e5440.

36. Wikoff, W. R.; Pendyala, G.; Siuzdak, G.; Fox, H. S. Metabolomic Analysis of the Cerebrospinal Fluid Reveals Changes in Phospholipase Expression in the CNS of SIV-Infected Macaques. J. Clin. Investig. 2008, 118, 2661-2669.

37. Tyburski, J. B.; Patterson, A. D.; Krausz, K. W.; Slavik, J.; Fornace, A. J. Gonzalez, F. J.; Idle, J. R. Radiation metabolomics. 1. Identification of Minimally Invasive Urine Biomarkers for $\gamma$-Radiation Exposure in Mice. Radiat. Res. 2008, 170, 1-14.

38. Soga, T.; Baran, R.; Suematsu, M.; Ueno, Y.; Ikeda, S.; Sakurakawa, T.; Kakazu, Y.; Ishikawa, T.; Robert, M.; Nishioka, T.; Tomita, M. Differen- 
tial Metabolomics Reveals Ophthalmic Acid as an Oxidative Stress Biomarker Indicating Hepatic Glutathione Consumption. J. Biol. Chemi. 2006, 281, 16768-16776.

39. Lin, Y.; Schiavo, S.; Orjala, J.; Vouros, P.; Kautz, R. Microscale LC-MSNMR Platform Applied to the Identification of Active Cyanobacterial Metabolites. Anal. Chem. 2008, 80, 8045-8054.

40. Trauger, S. A.; Kalisak, E.; Kalisiak, J.; Morita, H.; Weinberg, M. V.; Menon, A. L.; Poole, F. L.; Adams, M. W. W.; Siuzdak, G. Correlating the Transcriptome, Proteome, and Metabolome in the Environmental Adaptation of a Hyperthermophile. J. Proteome Res. 2008, 7, 1027-1035.

41. Tiller, P. R.; Yu, S.; Castro-Perez, J.; Fillgrove, K. L.; Baillie, T. A. High-Throughput, Accurate Mass Liquid Chromatography/Tandem Mass Spectrometry on a Quadrupole Time-of-Flight System as a 'FirstLine' Approach for Metabolite Identification Studies. Rapid Commun. Mass Spectrom. 2008, 22, 1053-1061.

42. Benton, H.; Wong, D.; Trauger, S.; Siuzdak, G. XCMS2: Processing Tandem Mass Spectrometry Data for Metabolite Identification and Structural Characterization. Anal. Chem. 2008, 80, 6382-6389.

43. Holsclaw, C. M.; Sogi, K. M.; Gilmore, S. A.; Schelle, M. W.; Leavell, M. D.; Bertozzi, C.R.; Leary, J. A. Structural Characterization of a Novel Sulfated Menaquinone produced by stf3 from Mycobacterium tuberculosis. ACS Chem. Biol. 2008, 3, 619-624.

44. Giavalisco, P.; Hummel, J.; Lisec, J.; Inostroza, A.C.; Catchpole, G.; Willmitzer, L. High-Resolution Direct Infusion-Based Mass Spectrometry in Combination with Whole C-13 Metabolome Isotope Labeling Allows Unambiguous Assignment of Chemical Sum Formulas. Anal. Chem. 2008, 80, 9417-9425.
45. Giavalisco, P.; Kohl, K.; Hummel, J.; Seiwert, B.; Willmitzer, L. C-13 Isotope-Labeled Metabolomes Allowing for Improved Compound Annotation and Relative Quantification in Liquid Chromatography-Mass Spectrometry-Based Metabolomic Research. Anal. Chem. 2009, 81, 65466551.

46. Hegeman, A. D.; Schulte, C. F.; Cui, Q.; Lewis, I. A.; Huttlin, E. L.; Eghbalnia, H.; Harms, A. C.; Ulrich, E. L.; Markley, J. L.; Sussman, M. R. Stable Isotope Assisted Assignment of Elemental Compositions for Metabolomics. Anal. Chem. 2007, 79, 6912-6921.

47. Hnatyshyn, S.; Sanders, M.; Shipkova, P.; Luk, E.; Warrack, B.; Reily, M. Automated Mass Spectra Interpretation Approach to Data Reduction for LC-MS Metabolomics Analysis. Proceedings of the ASMS Conference on Mass Spectrometry and Allied Topics; Denver, CO, June, 2008.

48. Draper, J.; Enot, D. P.; Parker, D.; Beckmann, M.; Snowdon, S.; Lin, W. C.; Zubair, H. Metabolite Signal Identification in Accurate Mass Metabolomics Data with MZedDB, an Interactive $\mathrm{m} / z$ Annotation Tool Utilizing Predicted Ionization Behavior 'Rules'. BMC Bioinformatics 2009, 10, 227.

49. Hochreiter, S.; Wagner, R. R. Bioinformatics Research and Development. Lecture notes in computer science. Proceedings of the First International Conference, BIRD 2007, Berlin, Germany, March, 2007. New York: Springer: xvi, p. 482

50. Kind, T.; Fiehn, O. Seven Golden Rules for Heuristic Filtering of Molecular Formulas Obtained by Accurate Mass Spectrometry. BMC Bioinformatics 2007, 8, 105.

51. Wren, J. D. A Global Meta-Analysis of Microarray Expression Data to Predict Unknown Gene Functions and Estimate the Literature-Data Divide. Bioinformatics 2009, 25, 1694-1701. 